\title{
Survival without Bronchopulmonary Dysplasia of Extremely Preterm Infants: A Predictive Model at Birth
}

\author{
Olivier Baud ${ }^{\mathrm{a}, \mathrm{b}}$ Matthew Laughon ${ }^{c}$ Philippe Lehert ${ }^{\mathrm{d}, \mathrm{e}}$ \\ ${ }^{a}$ Division of Neonatology and Pediatric Intensive Care, Children's University Hospital of Geneva and University \\ of Geneva, Geneva, Switzerland; 'bniversité Paris Diderot, Sorbonne Paris Cité, INSERM U1141, Paris, France; \\ 'University of North Carolina at Chapel Hill, Chapel Hill, NC, USA; ${ }^{\mathrm{d} F a c u l t y}$ of Medicine, University of Melbourne, \\ Melbourne, VIC, Australia; ${ }^{\mathrm{E}}$ Faculty of Economics, University of Louvain, Ottignies-Louvain-la-Neuve, Belgium
}

\section{Keywords}

Prediction model · Bronchopulmonary dysplasia · Risk .

Extremely preterm infants

\begin{abstract}
Introduction: Early prediction of survival without bronchopulmonary dysplasia (BPD) at 36 weeks of postmenstrual age remains challenging for infants born extremely preterm. We aimed to provide a new predictive model including variables available only at or soon after birth based on the literature and existing models. Methods: We conducted a systematic review to identify all variables considered to be significant predictors of BPD and survival at birth in extremely preterm infants. We then assessed the external validity of the Eunice Kennedy Shriver National Institute of Child Health and Human Development (NICHD) Neonatal Research Network BPD estimator on the PREMILOC cohort, a recent French study with a large sample of extremely preterm infants and a vast number of variables at baseline. Finally, we attempted to improve this model by testing the added value of other early predictors reported in previous studies. Results: Restricted to baseline predictors, the NICHD Neonatal Research Network BPD estimator confirmed its calibration and fair discrimination (area under the receiver operating characteristic
\end{abstract}

[auROC] $[95 \% \mathrm{Cl}]=0.73[0.68-0.77]$ when used with a published model and auROC $[95 \% \mathrm{Cl}]=0.77$ [0.73-0.81] when fitted to the PREMILOC dataset). We were able to improve the discriminatory power by adding candidate variables at birth associated with BPD in previous studies. The modified best predicting model included gestational age at birth, birthweight, respiratory support at baseline, gender, center effect, and multiple pregnancy as baseline predictors. This model showed significantly better discrimination (auROC $[95 \% \mathrm{Cl}]=0.85[0.82-0.88]$ ) and better confirmed calibration (Hosmer-Lemeshow test, $p=0.45$ ). Conclusion: This new model, based on 6 early predictors, appears to improve the prediction soon after birth of BPD-free survival in extremely preterm infants.

๑) 2021 S. Karger AG, Basel

\section{Introduction}

Bronchopulmonary dysplasia (BPD) is the most common chronic lung disease of infancy and a major cause of neurodevelopmental impairment, long-term breathing problems, and mortality $[1,2]$. Despite significant advances in neonatal intensive care, early prediction of $\mathrm{BPD}$ remains a challenge for the most preterm infants. Predic-
Correspondence to:

Olivier Baud, olivier.baud@ hcuge.ch 
tive models of BPD and survival based on characteristics of the neonate may be useful for estimating the probable hazard level. Several approaches to construct predictive models have been proposed based on various sets of predictors, measured both soon after birth and during follow-up. Hence, the Eunice Kennedy Shriver National Institute of Child Health and Human Development (NICHD) developed a BPD estimator, considered to be the best current tool to identify neonates at risk of developing BPD [3]. It has been externally validated using a limited number of cohorts [4] but not on a large European cohort of extremely preterm infants. In addition, Onland et al. [5] concluded, in a systematic review in 2013, that current predictive models cannot be easily used in clinical practice due to low statistical quality.

We aimed to validate the NICHD BPD estimator on a large cohort of extremely preterm infants recruited in France and revise this model using solely other potential early predictors available soon after birth and considered to be risk factors of BPD or death. This new model is expected to help clinicians accurately estimate the probability of BPD-free survival of extremely preterm infants at birth and could be particularly useful in at least 2 circumstances:

\section{Methods and Statistical Techniques}

\section{Literature Review}

A prespecified protocol was registered for this systematic literature review of the risk factors of BPD (PROSPERO 2019 CRD42019118715, https://www.crd.york. ac.uk/prospero/display_record.php? ID = CRD42019118715). Two independent reviewers (P.L. and O.B.) searched for publications in English from January 2000 to December 2020 in PubMed using specific Medical Subject Headings (MeSH terms) and keywords (see online suppl. Table 1; for all online suppl. material, see www.karger.com/doi/10.1159/000515898). We considered both interventional and observational studies but disregarded review articles and guidelines. We further limited our search to studies assessing variables possibly associated with BPD in preterm neonates. Finally, we searched "bronchopulmonary dysplasia AND risk factor" in PubMed to identify any other useful baseline predictors $(N=1,072)$. The PRISMA checklist for systematic review is available in the online suppl. material.

We assessed the risk of bias in each included observational study using the Strengthening the Reporting of Observational Studies in Epidemiology (STROBE) instrument and in each clinical trial using the Cochrane Collaboration tool [6], assigning an overall quality grade of high, medium, low, or uncertain to each selected publication. Eligible covariates to be used as parameters for the prognostic model were defined as any baseline characteristic supported by at least 1 high-quality clinical trial or at least 1 high-quality noninterventional study to have an impact on BPD.

\section{External Validation of the NICHD BPD Estimator}

We investigated the external validity of the NICHD BPD estimator as the best available predictive model using the PREMILOC French cohort. The PREMILOC trial [7] was a recent trial aiming to assess the efficacy and safety of hydrocortisone in the reduction of BPD and death in neonates. Based on a large sample size $(n=523)$ of extremely preterm infants and an extensive list of variables documented at baseline, this trial provided highquality material for external validation. We evaluated the discriminatory power of the model: area under the receiver operating characteristic (auROC) curve and calibration (Hosmer-Lemeshow [HL] test) and significance of the intercept and slope of the fitting line between observed and predicted values.

\section{Optimization of the NICHD BPD Estimator}

Based on our literature review, which identified the baseline variables available at birth associated with BPD or death, we attempted to upgrade the NICHD predictive model by testing the added value of variables that were considered to be potential predictors. We used a mixed linear model featuring logistic regression on the binary studied endpoint, allowing the combination of fixed and random covariates. Variables were sequentially tested by decreasing value of the observed effect size pooled across all studies that tested the given covariate and weighted by the inverse of its variance. At each iteration, the candidate variable entered the model when both a significant change in the deviance $\chi^{2}$ between nested designs and significance of the Net Reclassification Index (NRI, sum of the changes in sensitivity and specificity) or the Integrated Discrimination Improvement (IDI) were found [8]. A backward process tested the significance of the other existing predictors for each new identified predictor.

\section{Challenging the Optimized Model and Sensitivity Analyses}

The estimated marginal values of BPD-free survival were calculated using the optimized model according to gestational age for both male and female patients by separately visualizing the additional effect of birthweight, respiratory support at baseline (RSB), and multiple preg- 
Table 1. Expected contribution of possible predictors of BPD, according to the study design and effect size [9-11, 13-15, 17, 18, 20-27]

\begin{tabular}{|c|c|c|c|c|}
\hline Baseline characteristic & Authors & Study design & Sample size & $\begin{array}{l}\text { Effect size } \\
\text { (odds ratio [95\% CI]) }\end{array}$ \\
\hline \multirow[t]{4}{*}{ IUGR } & Bose et al. [13] & PC & 1,241 & $2.6[1.9-3.6]$ \\
\hline & Zeitlin et al. [15] & PC & 4,525 & $6.42[4.51-9.20]$ \\
\hline & Eriksson et al. [14] & $\mathrm{CC}$ & 2,255 & $2.7[2.1-3.6]$ \\
\hline & Klinger et al. [9] & PC & 12,139 & $2.6[2.2-3.1]$ \\
\hline \multirow{6}{*}{ Gestational age } & Zhang et al. [11] & $\mathrm{RC}$ & 3,538 & $9.5[2.6-34.7]$ \\
\hline & Klinger et al. [9] & PC & 12,139 & \\
\hline & $>30$ weeks & & & 1 \\
\hline & $28-29$ weeks & & & $3.6[3.0-4.3]$ \\
\hline & 26-27 weeks & & & $8.8[7.2-10.7]$ \\
\hline & $24-25$ weeks & & & $25.2[20.0-31.8]$ \\
\hline \multirow[t]{2}{*}{ Sex (male) } & Carmichael et al. [10] & RC & 12,621 & $1.3[1.2-2.0]$ \\
\hline & Klinger et al. [9] & PC & 12,139 & $1.4[1.3-1.6]$ \\
\hline Multiple pregnancy & Klinger et al. [9] & PC & 12,139 & $1.1[0.9-1.2]$ \\
\hline \multirow{2}{*}{ Gestational hypertension } & Yen et al. [18] & $\mathrm{RC}$ & 5,753 & $0.78[0.62-0.98]$ \\
\hline & Gemmell et al. [17] & $\mathrm{RC}$ & 27,846 & $1.16[1.05-1.27]$ \\
\hline \multirow[t]{4}{*}{ Antenatal steroids } & Melamed et al. [20] & $\mathrm{RC}$ & & \\
\hline & Singletons & & 6,950 & $0.8[0.6-1.0]$ \\
\hline & Twins & & 2,516 & $0.7[0.4-1.1]$ \\
\hline & McGoldrick et al. [21] & SR and MA & 745 & $0.9[0.4-1.8]$ \\
\hline \multirow[t]{4}{*}{ Chorioamnionitis } & Zhang et al. [11] & $\mathrm{RC}$ & 3,538 & $42[6-292]$ \\
\hline & Hartling et al. [22] & SR and MA & 15,295 & $1.6[1.1-2.2]$ \\
\hline & Torchin et al. [23] & PC & 2,513 & $0.9[0.5-1.8]$ \\
\hline & Villamor-Martinez et al. [24] & SR and MA & 244,096 & $1.3[1.2-1.4]$ \\
\hline \multirow[t]{5}{*}{ Ventilation } & Isayama et al. [25] & SR and MA & 4,455 & $0.5[0.4-0.9]$ (LISA vs. nasal CPAP) \\
\hline & Gagliardi et al. [27] & $\mathrm{RC}$ & 1,260 & \\
\hline & $\mathrm{MV}$ for $<24 \mathrm{~h}$ & & & $1.0[0.4-2.8]$ \\
\hline & $\mathrm{MV}$ for $24 \mathrm{~h}-7$ days & & & $2.4[1.1-5.3]$ \\
\hline & MV for $>7$ days & & & $14.9[6.6-33.4]$ \\
\hline Center effect & Lapcharoensap et al. [26] & $\mathrm{RC}$ & 15,779 & $\begin{array}{l}1.23[1.02-1.49] \text { for NICU II } \\
1.04[0.95-1.14] \text { for NICU III }\end{array}$ \\
\hline
\end{tabular}

$\mathrm{RC}$, retrospective cohort; PC, prospective cohort; CC, case control; SR and MA, systematic review and meta-analysis; BPD, bronchopulmonary dysplasia; IUGR, intrauterine growth restriction; LISA, low-invasive surfactant administration; MV, mechanical ventilation.

nancy. Finally, we conducted sensitivity analyses to assess the robustness of the model by (1) repeating the test of the optimized model in the PREMILOC trial testing the placebo group only, (2) comparing stepwise regression analysis and the backward strategy based on the complete list of predictors at baseline, (3) testing the optimized model considering the center as a fixed factor, and (4) testing the model on 2 subsets of patients, with and without severe adverse postnatal events, all risk factors for death or BPD occurring between baseline (birth) and the final endpoint (36 weeks of postmenstrual age [PMA]).

\section{Results}

\section{$B P D$ Predictive Variables at Birth: A Systematic} Review

The main goal of the literature review was to identify baseline characteristics soon after birth previously reported to be potential predictors of BPD. The terms/keywords used for this systematic review are summarized in online suppl. Table 1 and the flowchart shown in online suppl. Fig. 1. Most perinatal and neonatal variables easily available after birth were screened (Table 1). No studies 


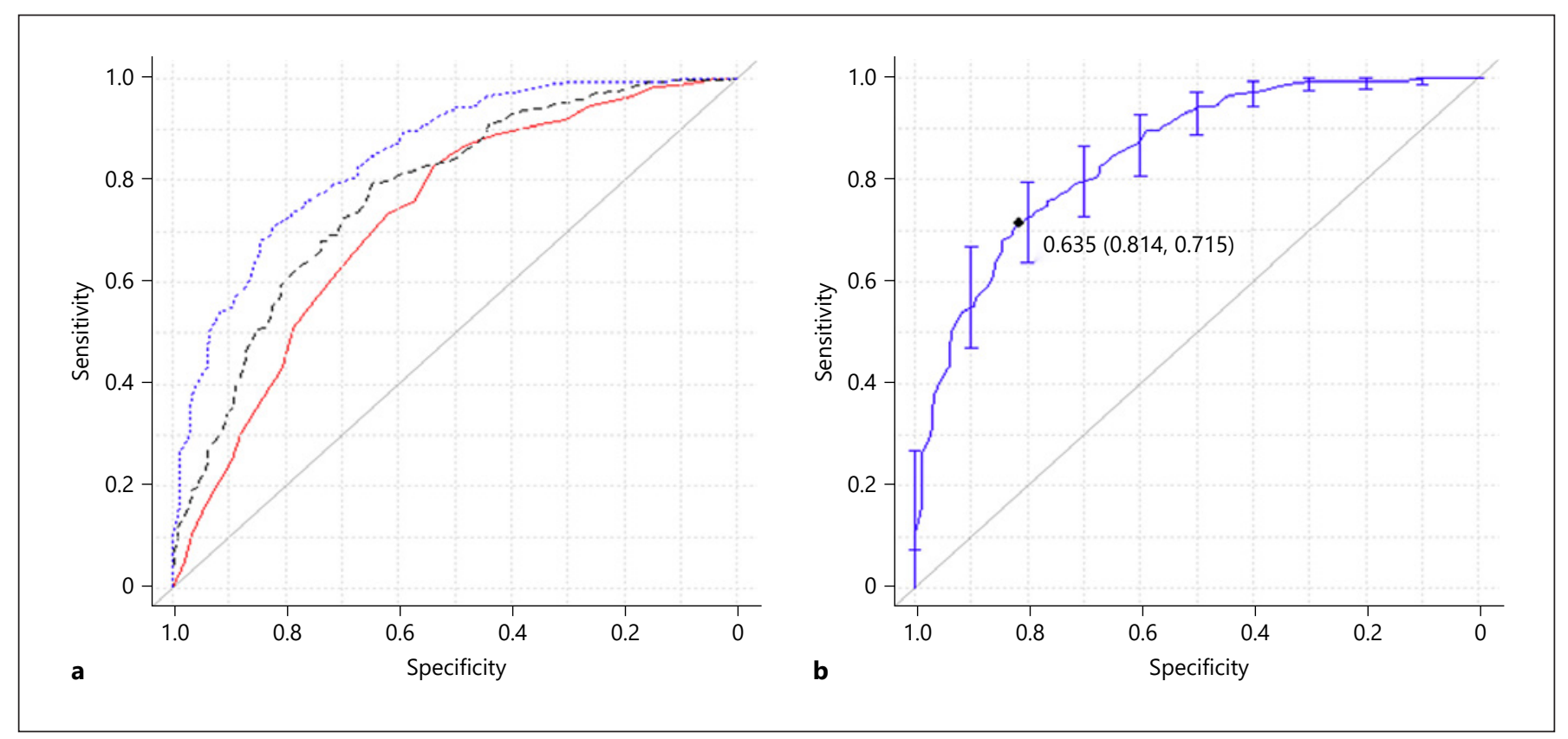

Fig. 1. Discriminative power of the initial and revised NICHD models. a auROC curves for the original NICHD model (red line, auROC [95\% CI] $=0.73[0.68-0.77]$ ), NICHD model fitted against the PREMILOC data (black line, auROC $[95 \% \mathrm{CI}]=0.77[0.73-0.81]$ ), and final revised model (blue line, auROC [95\% CI $]=0.85[0.82-0.88]$ ). b auROC curve for the final revised model with 95\% CI and Youden point, providing a specificity of 0.81 and a sensitivity of 0.72. NICHD, Eunice Kennedy Shriver National Institute of Child Health and Human Development; auROC, area under the receiver operating characteristic.

were found to definitively support the association of BPD with premature rupture of the membrane, tocolytics, gestational diabetes, mode of delivery, parental ethnicity, or delivery under general anesthesia. A large nationwide observational study showed an association between multiple gestation and BPD in preterm infants but only in an unadjusted comparison [9]. We identified multiple largescale high-quality studies supporting birthweight, gestational age, and sex (male gender) [10] as covariates associated with the risk of BPD $[9,11]$. Intrauterine growth restriction (IUGR) was found to be significantly associated with an increased risk of BPD [12-15] and altered pulmonary function in preterm infants [16]. Large-scale studies showed an association between the occurrence of BPD and gestational hypertension, an event frequently associated with IUGR $[17,18]$. Antenatal steroids were designated as a covariate, as they may affect lung maturity and the need for mechanical ventilation and thus indirectly affect the occurrence of BPD [19-21]. Chorioamnionitis was not selected as a relevant predictor because many studies reported conflicting conclusions and because the diagnostic criteria are not always available at birth [22-24].
Concerning early postnatal variables, the need for ventilation at birth and its duration were found to be associated with BPD $[27,28]$. These findings were also supported by the Bayesian random-effects network meta-analyses on ventilatory methods (30 clinical trials, 5,598 subjects) [25]. Finally, a recent high-quality study supported an association between the center and BPD occurrence [26].

A systematic review of the literature conducted by Onland et al. [5] identified 26 clinical prediction models for BPD. The NICHD BPD estimator is considered the best calibrated model [3] and includes the parameters gestational age, birthweight, race/ethnicity, sex, respiratory support, and fraction of inspired oxygen $\left(\mathrm{FiO}_{2}\right)$.

\section{Validation of the NICHD BPD Estimator on the PREMILOC Cohort}

We used the original formulation of the NICHD BPD estimator [3], separately on survival and the absence of $\mathrm{BPD}$, and intersected the results to show fair discrimination (auROC $[95 \% \mathrm{CI}]=0.73[0.68-0.77])$, with an acceptable calibration (HL test, $p=0.234$ ) (Fig. 1). We then fitted the PREMILOC dataset to the NICHD BPD estimator by classifying the level of RSB according to the highest 
Table 2. NICHD BPD estimator to predict BPD-free survival: logistic regression applied to the PREMILOC cohort

\begin{tabular}{lcr}
\hline Variable & Odds ratio (95\% CI) & $p$ value \\
\hline Overall (reference population*) & $0.62(1.08-2.50)$ & 0.021 \\
Gestational age (per week) & $1.43(1.08-1.87)$ & 0.011 \\
Birthweight (per gram) & $1.007(1.005-1.009)$ & $<0.001$ \\
Gender (female) & $2.92(1.86-4.59)$ & $<0.001$ \\
RSB** & $0.45(0.28-0.73)$ & 0.001 \\
$\quad$ Moderate & $0.13(0.06-0.26)$ & $<0.001$ \\
\hline
\end{tabular}

NICHD, Eunice Kennedy Shriver National Institute of Child Health and Human Development; BPD, bronchopulmonary dysplasia; RSB, respiratory support at baseline; $\mathrm{FiO}_{2}$, fraction of inspired oxygen. * Overall proportion of BPD-free survival for the reference population defined as male patients born at 26 weeks' gestation, birthweight of $850 \mathrm{~g}$, and mild respiratory support at baseline, defined as noninvasive ventilation with an $\mathrm{FiO}_{2}<30 \%$. ** RSB-moderate denotes respiratory support at baseline by mechanical ventilation with an $\mathrm{FiO}_{2}<30 \%$; RSB-severe denotes respiratory support at baseline by mechanical ventilation with an $\mathrm{FiO}_{2}$ $\geq 30 \%$. The effects of RSB-moderate and RSB-severe are compared to the reference of RSB-mild (noninvasive ventilation with an $\mathrm{FiO}_{2}$ $<30 \%)$.

required respiratory support before 24 postnatal hours into 3 categories: RSB-mild: noninvasive ventilation with an $\mathrm{FiO}_{2}<30 \%$; RSB-moderate: mechanical ventilation with an $\mathrm{FiO}_{2}<30 \%$; and RSB-severe: mechanical ventilation with an $\mathrm{FiO}_{2} \geq 30 \%$. The reference population for the model was defined as male patients born at 26 weeks of gestation, birthweight of $850 \mathrm{~g}$, and RSB-mild. The proportion of BPD-free surviving infants associated with this reference population was $61.9 \%$ (40.1-92.7). We found all terms included in the NICHD BPD estimator to be highly significant, except $\mathrm{FIO}_{2}$ (OR $[95 \% \mathrm{CI}]=0.94[0.82-$ 1.03 ] $p=0.124$ ) and the ethnic group (Caucasian vs. others, OR $[95 \% \mathrm{CI}]=1.03[0.68-1.58], p=0.89)$. Following the removal of these 2 variables, 4 significant parameters remained significantly predictive: gestational age, female sex, birthweight, and RSB, with a proportional decrease in the likelihood of BPD-free survival for RSB-moderate and RSB-severe infants (Table 2). We obtained significantly better discrimination using this new formulation (auROC $[95 \% \mathrm{CI}]=0.77[0.73-0.81]$ ) (Fig. 1), with a satisfactory calibration (HL test, $p=0.546$ ).

\section{Model Optimization and Sensitivity Analyses}

In the next step, we attempted to improve the determination of the NICHD BPD estimator using other baseline
Table 3. Test of possible additional predictors to increase the precision of the predictive model

\begin{tabular}{lrc}
\hline Variable & NRI $(p$ value $)$ & \multicolumn{1}{l}{$\begin{array}{l}\text { IDI } \\
(p \text { value })\end{array}$} \\
\hline Gestational hypertension & $-0.005(0.578)$ & $0(0.85)$ \\
Cesarean section & $0.009(0.687)$ & $0.01(0.09)$ \\
Antenatal steroids & $0.006(0.223)$ & $0.01(0.15)$ \\
Chorioamnionitis & $-0.013(0.257)$ & $0(0.6)$ \\
IUGR & $0.004(0.564)$ & $0(0.76)$ \\
Multiple pregnancy & $0.027(0.038)$ & $0.01(0.01)$ \\
All variables except multiple & & \\
\multicolumn{1}{c}{ pregnancy* } & $0.044(0.067)$ & $0.01(0.02)$ \\
\hline
\end{tabular}

The significance of the added predictive value of each covariate not yet entered into the model but identified as a predictor in the literature was assessed by NRI. NRI, Net Reclassification Improvement; IDI, Integrated Discrimination Improvement; IUGR, intrauterine growth restriction. ${ }^{*}$ Cumulative NRI and IDI in considering all candidate predictors together, except multiple pregnancy, which was already significant.

variables not yet included in the model but described as potential predictors in the literature (Table 1 ). We sequentially tested the predictive value of each variable in terms of BPD-free survival on the PREMILOC dataset. Chorioamnionitis, antenatal steroids, gestational hypertension, Cesarean section, and IUGR were not found to add significant predictive value and were discarded (Table 3). Only 2 predictors were identified with a significant added predictive value: multiple pregnancy and center, leading to a revised model (Table 4). Based on the above-described reference population, we found a virtually unchanged value for BPD-free survival of $68.1 \%$ (57.2-78.3) but strong center heterogeneity, with a standard deviation of $37.1 \%$ and a resulting confidence of BPD-free survival across centers from 48.1 to $83.4 \%$. Although all the existing terms remained unchanged, we observed a highly significant effect of multiple pregnancy (OR $[95 \% \mathrm{CI}]=0.50[0.32-$ $0.80], p<0.001)$. The optimized model was able to predict BPD-free survival with better discrimination (auROC $[95 \% \mathrm{CI}]=0.85[0.82-0.88])$ and a still acceptable calibration (HL test, $p=0.623$ ) (Fig. 1). The algorithm of this model is presented in the online suppl. material.

The estimated marginal values of BPD-free survival, as calculated using the optimized model, are shown in Figure 2, according to gestational age for both male and female patients, separately visualizing the additional effect of birthweight, RSB, and multiple pregnancy. Finally, we conducted sensitivity analyses to assess the robustness of 
Table 4. Final revised model to predict BPD-free survival

\begin{tabular}{llr}
\hline Variable & Odds ratio $(95 \% \mathrm{CI})$ & $p$ value \\
\hline Overall proportion of BPD survival* & $68.1 \%(57.2-78.3)$ & \\
Center effect** & $\mathrm{SD}=37.4 \%[48.1 \%, 83.4 \%]$ & $<0.001$ \\
Gestational age -26 (per week) & $1.474(1.11-1.943)$ & 0.001 \\
Birthweight - 850 (per gram) & $1.007(1.005-1.009)$ & $<0.001$ \\
Female gender & $2.744(1.740-4.327)$ & $<0.001$ \\
RSB*** & & 0.003 \\
Moderate & $0.449(0.275-0.731)$ & $<0.001$ \\
Severe & $0.128(0.064-0.256)$ & 0.001 \\
Multiple pregnancy & $0.504(0.319-0.796)$ & \\
\hline
\end{tabular}

$\mathrm{BPD}$, bronchopulmonary dysplasia; RSB, respiratory support at baseline; $\mathrm{FiO}_{2}$, fraction of inspired oxygen. * Intercept of the model estimating the proportion of BPD-free survival for the population defined as male patients born at 26 weeks' gestation with a birthweight of $850 \mathrm{~g}$ and mild respiratory support at baseline, defined as noninvasive ventilation with an $\mathrm{FiO}_{2}<30 \%$. ** Center effect measured by the standard deviation of the proportion of BPD, survival, and the resulting $95 \%$ confidence limits of the proportion of variation between centers. ${ }^{* * *} \mathrm{RSB}$-moderate denotes respiratory support at baseline by mechanical ventilation with an $\mathrm{FiO}_{2}<30 \%$; RSB-severe denotes respiratory support at baseline by mechanical ventilation with an $\mathrm{FiO}_{2} \geq 30 \%$. The effects of RSB-moderate and RSB-severe are compared to the reference of RSB-mild (noninvasive ventilation with an $\left.\mathrm{FiO}_{2}<30 \%\right)$.

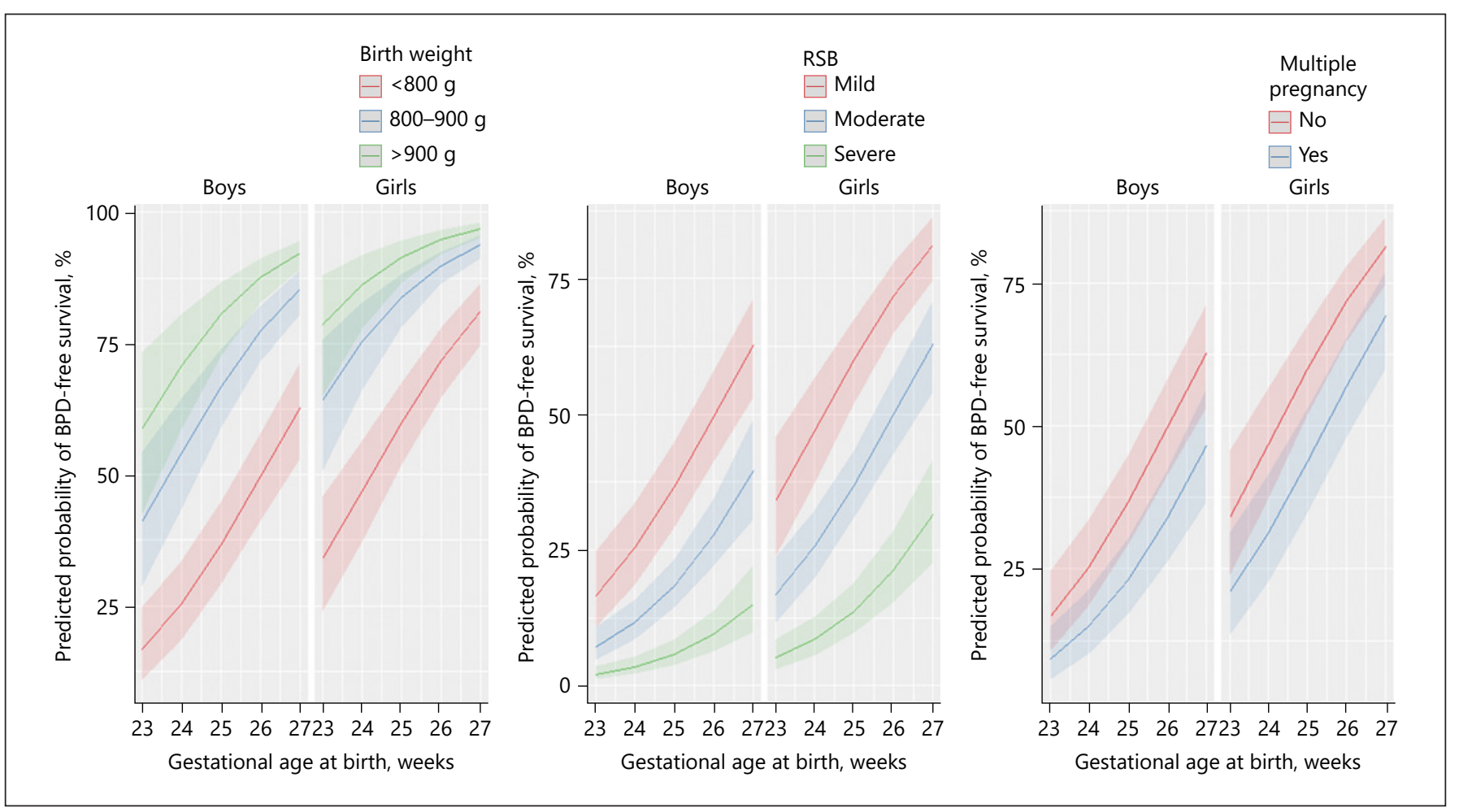

Fig. 2. Probability of BPD-free survival according to baseline predictors of BPD and gestational age, birthweight group, RSB, and multiple pregnancy, separately for boys and girls. RSB-mild: noninvasive ventilation with an $\mathrm{FiO}_{2}<30 \%$, RSB-moderate: respiratory mechanical ventilation with an $\mathrm{FiO}_{2}<30 \%$, RSB-severe: mechanical ventilation with an $\mathrm{FiO}_{2} \geq 30 \%$. BPD, bronchopulmonary dysplasia; RSB, respiratory support at baseline; $\mathrm{FiO}_{2}$, fraction of inspired oxygen. 
the model. (1) The optimized model was repeated in the PREMILOC trial testing the placebo group only $(n=264)$. All the terms remained similar and significant, with a mean relative error of $0.03 \%$. (2) Both stepwise regression analysis and the backward strategy based on the complete list of predictors at baseline yielded the same results, except for a significant effect of delivery by Cesarean section (OR $[95 \% \mathrm{CI}]=7.27[1.23-1,256], p=0.03)$. (3) The same results were obtained when considering the center as a fixed factor. (4) The model was tested in the subpopulation of patients who experienced at least 1 severe postnatal adverse event between birth and 36 weeks of PMA (including late-onset sepsis, PDA either medically treated or surgically closed, high-grade IVH, severe necrotizing enterocolitis, and spontaneous intestinal perforation). The prediction was lower in this subset of patients, with $68 \%$ well classified, a determination of 0.17 , and an auROC $=$ 0.76 . By contrast, our prediction reached $91 \%$ well classified, a determination of 0.43 , and an auROC $=0.91$ for patients without any severe postnatal events.

\section{Discussion/Conclusion}

Here, we updated the NICHD BPD estimator based on a systematic review of BPD risk factors and externally validated both the initial and revised models using the PREMILOC cohort of extremely preterm infants. In addition to baseline variables included in this original model, multiple pregnancies and a center effect, but not maternal ethnicity, were found to be significantly associated with the occurrence of BPD.

The NICHD BPD estimator [3] is generally considered to be the best model. The European validation of the NICHD BPD estimator is of interest because this model has been mainly tested in very preterm infants born in North America, where the incidence of BPD has been reported to be substantially different from that in Europe. We used a data-driven approach to optimize the BPD predictive model for surviving infants by selecting relevant baseline variables from the literature. Early after birth, an improved prediction of BPD would not only have a large impact on the classification of infants at risk of respiratory morbidities but also help select the besttargeted population that would benefit from a preventive strategy.

Our data confirm the key role of fetal growth at birth in the subsequent development of BPD and that RSB graded soon after birth is statistically associated with death or BPD at 36 weeks of PMA. RSB is an updated and clinically relevant combination of respiratory support and $\mathrm{FiO}_{2}$ derived from Sweet et al. [29].

The lack of a significant association between ethnic group and BPD-free survival contradicts the results of several studies [30]. This can be explained by differences between France and the USA in (1) the genetic variants related to lung development, drug metabolism, and immune response in high-risk premature infants; (2) the risk of prematurity-related complications according to ethnicity; and (3) access to health care depending on the ethnic group.

Concerning multiple gestation as a novel baseline variable, now included in the revised model, there has been an ongoing debate about differences in respiratory outcomes between very preterm singletons and very preterm twin infants $[9,31]$. The complex amalgam of mortality and morbidities in twins, associated with chorionicity and IUGR, may explain these differences.

We observed a significant center effect in the prediction of BPD-free survival, as previously reported. In particular, BPD rates in very preterm survivors varied widely across European regions, independently of mortality rates [32]. The magnitude of the center effect in the occurrence of BPD or death was large and persisted after correction for clinical covariates associated with BPD/ death [33]. Clustered and unmeasured variables are yet to be investigated to delineate the underlying factors responsible for the center effect on predicting BPD. This effect persisted in our study while interrelated factors, such as ventilatory support at birth, were also considered as covariates included in the model. However, further studies are needed to better delineate the role of medical/ nurse staffing, compliance with guidelines, and teaching activities in this center effect.

One limitation of this study is related to the external validation of the model, based on a large, recent, and very well-documented cohort of extremely preterm infants. Despite the large set of baseline variables collected in this trial, other variables related to the quality of perinatal care or early treatment may also influence the incidence of BPD. Although a substantial proportion of infants were found to still be misclassified using this new model, the increase in discrimination relative to the NICHD BPD estimator is significant (from an auROC of 0.78-0.85). The difference between these 2 values is much greater than it would appear. Indeed, the auROC is not very sensitive to increases in classification. Our model yielded $81 \%$ well-classified patients ( $91 \%$ for patients without severe adverse postnatal events), whereas the same value for the Laughon model was $69 \%$, leading to a clinically significant $17 \%$ improvement. 
In conclusion, we externally validated the NICHD BPD estimator on European data, and our revised version of the model improved the determination of predicting BPD-free survival of extremely preterm infants. This updated model, restricted to baseline variables, is expected to better predict the outcome of the infants at birth and therefore may provide a better selection of those who could benefit from preventive strategies.

\section{Acknowledgement}

The authors thank Aguettant Pharma for providing an unconditional grant. They thank Amel Ouslimani, Elodie Soler (Direction de la Recherche Clinique Et Du Développement, Assistance Publique-Hôpitaux de Paris, Paris, France), Prof. Corinne Alberti and Adyla Yacoubi (Unit of Clinical Epidemiology, Assistance Publique-Hôpitaux de Paris, Centre Hospitalier Universitaire Robert Debré) for their role in the PREMILOC trial.

\section{Statement of Ethics}

The PREMILOC trial used for external validation of the model was approved by the National Ethics Committee (Comité de Protection des Personnes [CPP], Ile-de-France II, Necker), the French National Drug Safety Agency (ANSM, EudraCT number 2007002041-20), and the French Data Protection Authority (Commis- sion Nationale de l'Informatique et des Libertés [CNIL]). Written informed consent was obtained from the parents of all eligible infants before randomization. The trial was registered at ClinicalTrials.gov (NCT00623740) before the first patient was enrolled.

\section{Conflict of Interest Statement}

The financial sponsor Aguettant Pharma had no role in the study design, data collection, analysis, decision to publish, or preparation of the manuscript.

\section{Funding Sources}

The PREMILOC trial was supported by a research grant from the French Ministry of Health. This study was supported by Assistance Publique-hôpitaux de Paris and Aguettant Pharma providing an unconditional grant.

\section{Author Contributions}

O.B. designed the study, wrote the first draft of the manuscript, and finalized the submitted version. M.L. revised and approved the final version of the manuscript. P.L. analyzed the data, revised the NICHD BPD estimator, and approved the final version of the manuscript.

\section{References}

1 Neubauer V, Junker D, Griesmaier E, Schocke M, Kiechl-Kohlendorfer U. Bronchopulmonary dysplasia is associated with delayed structural brain maturation in preterm infants. Neonatology. 2015;107(3):179-84.

2 Jobe AH, Bancalari EH. Controversies about the definition of bronchopulmonary dysplasia at 50 years. Acta Paediatr. 2017 May; 106(5):692-3.

3 Laughon MM, Langer JC, Bose CL, Smith PB, Ambalavanan N, Kennedy KA, et al. Prediction of bronchopulmonary dysplasia by postnatal age in extremely premature infants. Am J Respir Crit Care Med. 2011 Jun 15;183(12): 1715-22.

4 Cuna A, Liu C, Govindarajan S, Queen M, Dai $\mathrm{H}$, Truog WE. Usefulness of an online risk estimator for bronchopulmonary dysplasia in predicting corticosteroid treatment in infants born preterm. J Pediatr. 2018 Jun; 197:23-e2.

5 Onland W, Debray TP, Laughon MM, Miedema M, Cools F, Askie LM, et al. Clinical prediction models for bronchopulmonary dysplasia: a systematic review and external validation study. BMC Pediatr. 2013 Dec 17;13: 207.

6 Higgins JP, Altman DG, Gøtzsche PC, Jüni P, Moher D, Oxman AD, et al. The Cochrane Collaboration's tool for assessing risk of bias in randomised trials. BMJ. 2011 Oct 18;343: d5928.

7 Baud O, Maury L, Lebail F, Ramful D, El Moussawi F, Nicaise C, et al. Effect of early low-dose hydrocortisone on survival without bronchopulmonary dysplasia in extremely preterm infants (PREMILOC): a doubleblind, placebo-controlled, multicentre, randomised trial. Lancet. 2016 Apr 30; 387(10030):1827-36.

8 Pencina MJ, D'Agostino RB Sr, D'Agostino RB Jr, Vasan RS. Evaluating the added predictive ability of a new marker: from area under the ROC curve to reclassification and beyond. Stat Med. 2008 Jan 30;27(2):157-12. discussion 207-12.

9 Klinger G, Sokolover N, Boyko V, Sirota L, Lerner-Geva L, Reichman B, et al. Perinatal risk factors for bronchopulmonary dysplasia in a national cohort of very-low-birthweight infants. Am J Obstet Gynecol. 2013 Feb; 208(2):115-9.

10 Carmichael SL, Kan P, Gould JB, Stevenson DK, Shaw GM, Lee HC. Maternal prepregnancy body mass index and risk of bronchopulmonary dysplasia. Pediatr Res. 2017 Jul; 82(1):8-13.

11 Zhang H, Fang J, Su H, Chen M. Risk factors for bronchopulmonary dysplasia in neonates born at $</=1500 \mathrm{~g}$ (1999-2009). Pediatr Int. 2011 Dec;53(6):915-20.

12 Monier I, Ancel PY, Ego A, Guellec I, Jarreau $\mathrm{PH}$, Kaminski M, et al. Gestational age at diagnosis of early-onset fetal growth restriction and impact on management and survival: a population-based cohort study. BJOG. 2017 Nov; 124(12):1899-906.

13 Bose C, Van Marter LJ, Laughon M, O'Shea TM, Allred EN, Karna P, et al. Fetal growth restriction and chronic lung disease among infants born before the 28th week of gestation. Pediatrics. 2009 Sep;124(3):e450-8.

14 Eriksson L, Haglund B, Odlind V, Altman M, Ewald U, Kieler H. Perinatal conditions related to growth restriction and inflammation are associated with an increased risk of bronchopulmonary dysplasia. Acta Paediatr. 2015 Mar;104(3):259-63.

15 Zeitlin J, El Ayoubi M, Jarreau PH, Draper ES, Blondel B, Kunzel W, et al. Impact of fetal growth restriction on mortality and morbidity in a very preterm birth cohort. J Pediatr. 2010 Nov; 157(5):733-9e1.

16 Ronkainen E, Dunder T, Kaukola T, Marttila $\mathrm{R}$, Hallman M. Intrauterine growth restriction predicts lower lung function at school age in children born very preterm. Arch Dis Child Fetal Neonatal Ed. 2016 Sept;101(5):F412-7. 
17 Gemmell L, Martin L, Murphy KE, Modi N, Håkansson S, Reichman B, et al. Hypertensive disorders of pregnancy and outcomes of preterm infants of 24 to 28 weeks' gestation. J Perinatol. 2016 Dec;36(12):1067-72.

18 Yen TA, Yang HI, Hsieh WS, Chou HC, Chen CY, Tsou KI, et al. Preeclampsia and the risk of bronchopulmonary dysplasia in VLBW infants: a population based study. PLoS One. 2013;8(9):e75168

19 Gagliardi L, Bellù R, Rusconi F, Merazzi D, Mosca F. Antenatal steroids and risk of bronchopulmonary dysplasia: a lack of effect or a case of over-adjustment? Paediatr Perinat Epidemiol. 2007 Jul;21(4):347-53.

20 Melamed N, Shah J, Yoon EW, Pelausa E, Lee SK, Shah PS, et al. The role of antenatal corticosteroids in twin pregnancies complicated by preterm birth. Am J Obstet Gynecol. 2016 Oct;215(4):482 e1-9.

21 McGoldrick E, Stewart F, Parker R, Dalziel SR. Antenatal corticosteroids for accelerating fetal lung maturation for women at risk of preterm birth. Cochrane Database Syst Rev. 2020 Dec;2512:CD004454.

22 Hartling L, Liang Y, Lacaze-Masmonteil T. Chorioamnionitis as a risk factor for bronchopulmonary dysplasia: a systematic review and meta-analysis. Arch Dis Child Fetal Neonatal Ed. 2012 Jan;97(1):F8-F17.
23 Torchin H, Lorthe E, Goffinet F, Kayem G, Subtil D, Truffert P, et al. Histologic chorioamnionitis and bronchopulmonary dysplasia in preterm infants: the epidemiologic study on low gestational ages 2 cohort. J Pediatr. 2017 Aug; 187:98-e3.

24 Villamor-Martinez E, Álvarez-Fuente $M$, Ghazi AMT, Degraeuwe P, Zimmermann LJI, Kramer BW, et al. Association of chorioamnionitis with bronchopulmonary dysplasia among preterm infants: a systematic review, meta-analysis, and metaregression. JAMA Netw Open. 2019 Nov 1;2(11):e1914611.

25 Isayama $\mathrm{T}$, Iwami $\mathrm{H}, \mathrm{McD}$ onald $\mathrm{S}$, Beyene J. Association of noninvasive ventilation strategies with mortality and bronchopulmonary dysplasia among preterm infants: a systematic review and meta-analysis. JAMA. 2016 Aug 9;316(6):611-24.

26 Lapcharoensap W, Gage SC, Kan P, Profit J, Shaw GM, Gould JB, et al. Hospital variation and risk factors for bronchopulmonary dysplasia in a population-based cohort. JAMA Pediatr. 2015 Feb;169(2):e143676.

27 Gagliardi L, Bellu R, Lista G, Zanini R, Network Neonatale Lombardo Study Group . Do differences in delivery room intubation explain different rates of bronchopulmonary dysplasia between hospitals? Arch Dis Child Fetal Neonatal Ed. 2011 Jan;96(1):F30-5.
28 Jensen EA, DeMauro SB, Kornhauser M, Aghai ZH, Greenspan JS, Dysart KC. Effects of multiple ventilation courses and duration of mechanical ventilation on respiratory outcomes in extremely low-birth-weight infants. JAMA Pediatr. 2015 Nov;169(11):1011-7.

29 Sweet DG, Carnielli V, Greisen G, Hallman M, Ozek E, Te Pas A, et al. European consensus guidelines on the management of respiratory distress syndrome: 2019 update. Neonatology. 2019;115(4):432-50.

30 Janevic T, Zeitlin J, Auger N, Egorova NN, Hebert P, Balbierz A, et al. Association of race/ethnicity with very preterm neonatal morbidities. JAMA Pediatr. 2018 Nov 1; 172(11):1061-9.

31 Papiernik E, Zeitlin J, Delmas D, Blondel B, Künzel W, Cuttini M, et al. Differences in outcome between twins and singletons born very preterm: results from a population-based European cohort. Hum Reprod. 2010 Apr;25(4): 1035-43.

32 Edstedt Bonamy AK, Zeitlin J, Piedvache A, Maier RF, van Heijst A, Varendi H, et al. Wide variation in severe neonatal morbidity among very preterm infants in European regions. Arch Dis Child Fetal Neonatal Ed. 2019 Jan; 104(1):F36-45

33 Ambalavanan N, Walsh M, Bobashev G, Das A, Levine B, Carlo WA, et al. Intercenter differences in bronchopulmonary dysplasia or death among very low birth weight infants. Pediatrics. 2011 Jan;127(1):e106-16. 